\title{
PERFIL EPIDEMIOLÓGICO E DISTRIBUIÇÃO ESPACIAL DA HANSENÍASE EM PAULO AFONSO, BAHIA
}

\author{
EPIDEMIOLOGICAL PROFILE AND SPATIAL \\ DISTRIBUTION OF LEPROSY IN PAULO AFONSO, BAHIA
}

\section{PERFIL EPIDEMIOLÓGICO Y DISTRIBUCION ESPACIAL DE LA LEPRA EN PAULO AFONSO, BAHÍA}

\author{
Yasmin Pereira Azevedo ${ }^{1}$ \\ Vitor Augusto da Silva Bispo ${ }^{2}$ \\ Ronaldo Inácio de Oliveira ${ }^{3}$ \\ Bruno Bezerra Gondim ${ }^{4}$ \\ Sélton Diniz dos Santos ${ }^{5}$ \\ Márcio Santos da Natividade ${ }^{6}$ \\ Joilda Silva Nery ${ }^{7}$
}

Como citar este artigo: Azevedo YP, Bispo VAS, Oliveira RI, Gondim BB, Santos SD, Natividade MS, et al. Perfil epidemiológico e distribuição espacial da hanseníase em Paulo Afonso, Bahia. Rev baiana enferm. 2021;35:e37805.

Objetivos: analisar as características epidemiológicas e distribuição dos casos novos de hanseníase na população de Paulo Afonso, Bahia, entre 2000 e 2015. Método: estudo descritivo com todos os casos novos de hanseníase notificados no Sistema de Informações de Agravos de Notificação. Foram utilizadas variáveis sociodemográficas e clínicas e calculados os coeficientes de detecção anuais. Estimativas populacionais foram obtidas no Instituto Brasileiro de Geografia e Estatística. Resultados: a maioria dos 1.069 casos novos notificados foi do sexo feminino (57,2\%), residiam na zona urbana (92\%), raça/cor parda $(66,92 \%)$, ensino fundamental $(67,6 \%)$, maiores de quinze anos $(92,5 \%)$, forma clínica tuberculoide $(48,26 \%)$, paucibacilares $(62,3 \%)$, grau de incapacidade física no diagnóstico $(45,93 \%)$ e na cura (29,75\%). Conclusão: o perfil epidemiológico da hanseníase em Paulo Afonso é similar ao nacional, houve variações nos coeficientes de detecção da hanseníase entre os anos e a análise espacial evidenciou distribuição heterogênea, com maior concentração de casos nos bairros periféricos.

Descritores: Doenças Transmissíveis. Saúde Pública. Hanseníase. Epidemiologia Descritiva. Análise Espacial.

Objectives: to analyze the epidemiological characteristics and distribution of new leprosy cases in the population of Paulo Afonso, Bahia, between 2000 and 2015. Method: descriptive study with all new cases of leprosy reported at the Notifiable Diseases Information System. Sociodemographic and clinical variables were used and annual detection coefficients were calculated. Population estimates were obtained from the Brazilian Institute of Geography and Statistics. Results: most of the 1,069 new reported cases were female (57.2\%), living in the urban area (92\%), brown race/color (66.92\%), elementary school (67.6\%), over fifteen years of age (92.5\%), tuberculoid clinical form (48.26\%), paucibacillary (62.3\%), degree of physical disability at diagnosis (45.93\%) and cure (29.75\%). Conclusion: the epidemiological profile of leprosy in Paulo Afonso is similar to the national one, with variations in leprosy detection

Estudante de Medicina. Universidade Federal do Vale do Rio São Francisco. Paulo Afonso, Bahia, Brasil. http://orcid.org/0000-0002-657/-4085.

Estudante de Medicina. Universidade Federal do Vale do Rio São Francisco. Paulo Afonso, Bahia, Brasil. http://orcid.org/0000-0002-4I I 6-7363

Estudante de Medicina. Universidade Federal do Vale do Rio São Francisco. Paulo Afonso, Bahia, Brasil. http://orcid.org/0000-0002-57/ 3-7556.

Estudante de Medicina. Universidade Federal do Vale do Rio São Francisco. Paulo Afonso, Bahia, Brasil. http://orcid.org/0000-000 I-9825-977।

Enfermeiro. Mestre em Saúde Coletiva. Docente da Universidade Estadual de Feira de Santana. Feira de Santana, Bahia, Brasil. http://orcid.org/0000-00023992-4353.

Bacharel em Ciências Biológicas. Doutor em Saúde Pública. Docente da Universidade Federal da Bahia. Salvador, Bahia, Brasil. marcio.natividade@outlook.com. http://orcid.org/0000-0002-3986-5656.

Biomédica. Doutora em Saúde Coletiva. Docente da Universidade Federal da Bahia. Salvador, Bahia, Brasil. http://orcid.org/0000-0002-I576-64I8. 
coefficients between years and spatial analysis showing heterogeneous distribution, with a higher concentration of cases in peripheral neighborhoods.

Descriptors: Communicable Diseases. Public Health. Leprosy. Descriptive Epidemiology. Spatial Analysis.

Objetivos: analizar las características epidemiológicas y la distribución de nuevos casos de lepra en la población de Paulo Afonso, Bahía, entre 2000 y 2015. Método: estudio descriptivo con todos los nuevos casos de lepra reportados en el Sistema de Información de Enfermedades Notificables. Se utilizaron variables sociodemográficas y clinicas y se calcularon los coeficientes anuales de detección. Las estimaciones de población se obtuvieron del Instituto Brasileño de Geografía y Estadística. Resultados: la mayoría de los 1.069 nuevos casos notificados fueron mujeres (57,2\%), que viven en el área urbana (92\%), raza/color parda (66,92\%), escuela primaria (67,6\%), con más de quince años (92,5\%), forma clinica de tuberculoide (48,26\%), paucibacilario (62,3\%), grado de discapacidad física al diagnóstico (45,93\%) y en la cura (29,75\%). Conclusión: el perfil epidemiológico de la lepra en Paulo Afonso es similar al nacional, hubo variaciones en los coeficientes de detección de lepra entre años y el análisis espacial mostró una distribución heterogénea, con una mayor concentración de casos en barrios periféricos.

Descriptores: Enfermedades transmisibles. Salud Pública. Lepra. Epidemiología descriptiva. Análisis espacial.

\section{Introdução}

A hanseníase é uma doença infectocontagiosa crônica, curável e de notificação compulsória que acomete pele, mucosas e nervos ${ }^{(1)}$. Manifesta-se neurodermatologicamente por meio de lesões de pele com graus de alteração de sensibilidade $^{(2)}$. A progressão da neuropatia pode desencadear incapacidades e deformidades físicas ${ }^{(1)}$ em consequências da não precocidade do diagnóstico e tratamento ${ }^{(3)}$.

Essa doença persiste como um problema de saúde pública no mundo com forte determinação social, pois a distribuição espacial possui íntima associação com as condições socioeconômicas das populações afetadas, ocorrendo uma tendência na concentração dos casos em estratos da sociedade menos favorecidos $^{(3)}$. A propagação da doença parece ser influenciada por fatores individuais e coletivos, como sexo, idade, suscetibilidade genética, condições socioeconômicas e geográficas; apesar dos esforços realizados, ainda são numerosos os casos de hanseníase em alguns países subdesenvolvidos ${ }^{(4)}$.

A Organização Mundial de Saúde (OMS), desde a década de 1990, propõe a integração das ações de controle da hanseníase ${ }^{(5)}$. No mesmo período, o Brasil iniciou esse processo, com a consolidação do Sistema Único de Saúde (SUS) e tendo a atenção primária à saúde como estratégia para reorientação dos modelos de atenção à saúde e de enfrentamento das desigualdades sociais em saúde ${ }^{(6)}$. No entanto, no ano 2000, houve a ampliação do escopo de ações e competências no âmbito municipal para o controle da hanseníase ${ }^{(7)}$.

O Brasil é atualmente o segundo país mais endêmico do mundo, tendo apresentado um alto coeficiente de detecção (12,2 casos a cada 100 mil habitantes) ${ }^{(4)}$, regular percentual de cura entre os casos novos entre 2012 e $2016^{(8)}$ e o maior número de casos novos ocorreu em homens, de cor parda, analfabetos ou com o ensino médio incompleto ${ }^{(4)}$. A região Nordeste, assim como as regiões Norte e Centro-Oeste, é a que possui maior concentração de casos de todo o país ${ }^{(4)}$. Sendo assim, as regiões com maior risco de se contrair a doença ${ }^{(4)}$ chegam a uma incidência cinco a oito vezes maior que as regiões Sudeste e Sul ${ }^{(8)}$.

Assim como no Brasil, o perfil socioeconômico dos casos no estado da Bahia, considerada área endêmica e maior estado do Nordeste, é semelhante ao perfil dos casos em todo o Brasil. Em 2017, o coeficiente de detecção foi classificado como alto (14,5 casos por $100 \mathrm{mil} \mathrm{ha-}$ bitantes) e 79,4\% dos casos obtiveram cura ${ }^{(9)}$. Ainda, o maior número de casos novos da hanseníase nesse estado ocorre em pessoas do sexo masculino, cor parda, analfabeto ou ensino fundamental incompleto, idade entre 30 e $44 \operatorname{anos}^{(10)}$. 
Paulo Afonso é destacado como um dos 20 municípios prioritários para ações de vigilância e controle da hanseníase no estado ${ }^{(11)}$. É considerado um dos clusters de alto risco para detecção de casos novos ${ }^{(12)}$. Há carência de estudos que abordem a situação da doença no município. Deste modo, a caracterização epidemiológica da hanseníase na cidade de Paulo Afonso por um período de 15 anos é de enorme importância para avaliar como está ocorrendo a abordagem diagnóstica, a efetividade do tratamento e o grau de incapacidades detectadas em uma cidade polo da Mesorregião do Vale do São Francisco.

O objetivo deste estudo foi analisar as características epidemiológicas e distribuição dos casos novos de hanseníase na população de Paulo Afonso, Bahia, entre 2000 e 2015.

\section{Método}

Trata-se de um estudo epidemiológico descritivo com base nos dados secundários provenientes do Sistema de Informações de Agravos de Notificação (SINAN). Analisou-se todos os casos novos de hanseníase diagnosticados e notificados entre 2000 e 2015 de residentes no município de Paulo Afonso.

Paulo Afonso é uma cidade baiana situada no Vale do São Francisco, com área de 1.579,723 km² e uma população aproximada de 120 mil habitantes ${ }^{(13)}$. Essa cidade é a segunda maior do Vale do São Francisco da Bahia e a maior da microrregião da qual faz parte.

Considerando a ficha de notificação referente ao município de residência e modo de entrada, foram excluídos deste estudo os casos de transferência do mesmo município (outra unidade), transferência de outro município (mesma unidade federativa), transferência de outro estado, transferência de outro país, recidiva, outros reingressos e ignorado.

O banco dados SINAN-Hanseníase foi disponibilizado pelo Ministério da Saúde (MS) em formato de planilha eletrônica do Microsoft Excel 2010. A extração dos dados do município de Paulo Afonso foi realizada em dezembro de 2017. As variáveis sociodemográficas foram as seguintes: idade ( $<15$ anos e $\geq 15$ anos), sexo (masculino e feminino), zona de residência (rural e urbana), raça/cor (branca, preta, parda, amarela e indígena), escolaridade (analfabeto, ensino fundamental, ensino médio e ensino superior). Já as variáveis clínicas consistiram na forma clínica (indeterminada, tuberculoide, dimorfa, virchowiana e não classificada), classificação operacional (paucibacilar e multibacilar), grau de incapacidade física (GIF) no diagnóstico (GIF 0, GIF I, GIF II, não avaliados) e GIF na cura (GIF 0, GIF I, GIF II, não avaliados e ignorados).

A análise dos dados foi realizada no primeiro semestre de 2018. Para as variáveis categóricas, foram calculadas as frequências absolutas e relativas. Para a variável contínua (idade) também foram calculadas médias anuais e desvio padrão. O cálculo dos coeficientes de detecção da hanseníase teve por base o número de casos e as estimativas populacionais de cada ano do estudo. As estimativas populacionais foram obtidas junto ao Instituto Brasileiro de Geografia e Estatística $(\mathrm{IBGE})^{(13)}$. Todos os parâmetros para a classificação dos indicadores de endemia no município de Paulo Afonso foram definidos pelo MS, na Portaria $\mathrm{n}^{\mathrm{o}}$ 149, de 3 de fevereiro de $2016^{(14)}$. $\mathrm{Na}$ análise, considerou-se o período de 2000 a 2015, em virtude da disponibilidade e completude dos dados no SINAN, principalmente na variável do encerramento dos casos. O ano 2000 foi o marco para a descentralização das ações de controle da hanseníase e melhoria da qualidade dos dados registrados.

Os endereços residenciais contidos nas fichas de notificação tornaram possível a identificação das localizações geográficas (latitude e longitude). As coordenadas foram capturadas em arquivo .dbf (dBase file) para a construção de mapas temáticos das áreas de incidência da hanseníase durante os anos de 2000 a 2015. Os arquivos shape foram extraídos do site do IBGE. Estes mapas possibilitaram a identificação de padrões de distribuição espacial da hanseníase em Paulo Afonso (BA), bem como a observação de clusters bem definidos.

Os dados gerados foram organizados em gráficos e tabelas, utilizando-se o Stata (versão 
13). Para o mapeamento, as coordenadas foram obtidas no Google Earth Pro. A construção dos mapas temáticos com as distribuições espaciais foi possibilitada pelo programa Qgis 2.18.14.

O presente estudo apresenta a síntese dos principais resultados do projeto de iniciação científica intitulado "Situação Epidemiológica da Hanseníase e Caracterização dos Casos Ocorridos no Município de Paulo Afonso - Bahia no período de 2000 a 2015", aprovado no Comitê de Ética em Pesquisa da Universidade Federal do Vale do São Francisco (UNIVASF), Parecer $n^{\circ} 2.785 .621$.

\section{Resultados}

Entre 2000 e 2015, foram notificados, em Paulo Afonso, 1.069 casos da doença. No geral, houve uma discreta redução do número absoluto de casos novos de hanseníase no município, comparando-se ao longo dos anos (39 casos em 2000 e 34 em 2015). Observou-se maior ocorrência de casos novos em $2004^{(127)}$ e em $2005^{(118)}$; e menor, nos anos de 2014 e de 2015, com 34 casos cada. A média foi de $66,8 \pm 29,2$ casos ao ano.

Em relação às características sociodemográficas dos casos novos, houve maior frequência no sexo feminino $(611 / 57,16 \%)$, cor da pele parda $(609 / 66,92 \%)$, níveis de escolaridade mais baixos (766/83,70\%) e entre jovens e adultos em idade economicamente ativa (989/92,50\%). A faixa etária com maior número de casos novos notificados foi de 21 a 30 anos (191/17,86\%). Enquanto a média de idade entre os casos novos

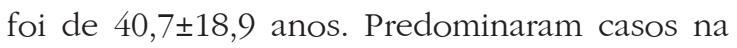
região urbana $(983 / 93,97 \%)$ (Tabela 1$)$.

Tabela 1 - Características sociodemográficas e clínicas dos casos novos de hanseníase. Paulo Afonso, Bahia, Brasil - 2000-2015. (N=1.069)

\begin{tabular}{|c|c|c|}
\hline Variáveis & $\mathbf{n}$ & $\%$ \\
\hline \multicolumn{3}{|l|}{ Sexo } \\
\hline Feminino & 611 & 57,16 \\
\hline Masculino & 458 & 42,84 \\
\hline \multicolumn{3}{|l|}{ Zona da moradia** } \\
\hline Urbana & 983 & 93,97 \\
\hline Rural & 63 & 6,02 \\
\hline \multicolumn{3}{|l|}{ Raça/cor* (2) } \\
\hline Branca & 220 & 24,17 \\
\hline Preta & 64 & 7,03 \\
\hline Amarela & 10 & 1,09 \\
\hline Parda & 609 & 66,92 \\
\hline Indígena & 7 & 0,76 \\
\hline \multicolumn{3}{|l|}{ Escolaridade* ${ }^{(3)}$} \\
\hline Analfabeto & 90 & 9,83 \\
\hline Ensino fundamental & 676 & 73,87 \\
\hline Ensino médio & 142 & 15,51 \\
\hline Ensino superior & 7 & 0,76 \\
\hline \multicolumn{3}{|l|}{ Idade } \\
\hline$<15$ anos & 80 & 7,48 \\
\hline$\geq 15$ anos & 989 & 92,50 \\
\hline \multicolumn{3}{|l|}{ Forma Clínica } \\
\hline Indeterminada & 124 & 11,60 \\
\hline Tuberculoide & 516 & 48,27 \\
\hline Dimorfa & 247 & 23,11 \\
\hline Virchowiana & 95 & 8,89 \\
\hline
\end{tabular}


Tabela 1 - Características sociodemográficas e clínicas dos casos novos de hanseníase. Paulo Afonso, Bahia, Brasil - 2000-2015. (N=1.069)

\begin{tabular}{|c|c|c|}
\hline Variáveis & $\mathbf{n}$ & $\%$ \\
\hline \multicolumn{3}{|l|}{ Forma Clínica } \\
\hline Não classificado & 12 & 1,12 \\
\hline Ignorados & 75 & 7,02 \\
\hline \multicolumn{3}{|c|}{ Classificação Operacional } \\
\hline Paucibacilar & 666 & 62,30 \\
\hline Multibacilar & 403 & 37,70 \\
\hline \multicolumn{3}{|c|}{ GIF no Diagnóstico } \\
\hline Grau 0 & 309 & 28,91 \\
\hline Grau 1 & 491 & 45,93 \\
\hline Grau 2 & 223 & 20,86 \\
\hline Não Avaliado & 46 & 4,30 \\
\hline \multicolumn{3}{|l|}{ GIF na Cura } \\
\hline Grau 0 & 310 & 29,00 \\
\hline Grau 1 & 318 & 29,75 \\
\hline Grau 2 & 126 & 11,79 \\
\hline Não Avaliado & 27 & 2,53 \\
\hline Ignorados & 288 & 26,94 \\
\hline
\end{tabular}

Fonte: Elaboração própria.

* Porcentagem calculada considerando apenas os indivíduos com informações para estas variáveis.

${ }^{(1)}$ Em 2,15\% (23 pessoas) das fichas essa variável não foi preenchida

(2) Em 15,56\% (159 pessoas) das fichas essa variável não foi preenchida.

(3) Em 14,95\% (154 pessoas) das fichas essa variável não foi preenchida.

Sobre as características clínicas, prevaleceram as formas clínicas e classificação operacional tuberculoide $(516 / 48,26 \%)$ e paucibacilar (666/62,30\%), respectivamente. Em relação ao GIF, a maioria dos casos já apresentava alguma GIF diagnóstico (714/66,67\%) e na cura (444/41,54\%).
A análise comparativa dos coeficientes de detecção de hanseníase geral em menores de 15 anos e maiores de 15 anos possibilitou observar-se que houve variações entre os anos. Os maiores coeficientes de detecção foram registrados em 2004 para todos os grupos (Gráfico 1).

Gráfico 1 - Coeficiente de detecção da hanseníase (por 100 mil habitantes) na população geral, maiores de 15 anos e menores de 15 anos. Paulo Afonso, Bahia, Brasil - 2000-2015

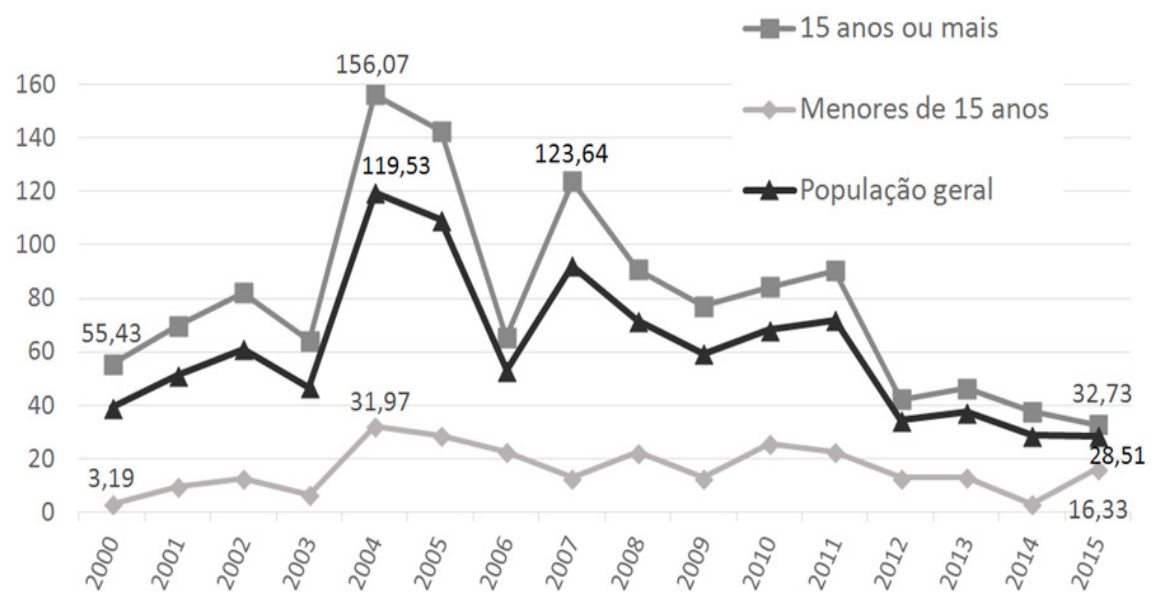

Fonte: Elaboração própria 
O coeficiente na população geral inicial e final foi de 39,10/100 mil habitantes e 28,5/100 mil habitantes, respectivamente. A média para o período de 2000 a 2015 foi de 60,80/100 mil habitantes. No ano de 2015, registrou-se o menor coeficiente de detecção (28,51/100 mil habitantes). A queda teve seu momento mais acentuado entre os anos de 2011 e 2012 e alterou a classificação da endemia na cidade para os grupos citados - de hiperendêmica para muito alta, parâmetro alcançado anteriormente apenas em 2000.

Ainda na população geral, tal indicador alcançou o valor de 119,53 casos por 100 mil habitantes, número três vezes maior do que o limite inferior para a classificação hiperendêmica. Esse também foi o ano com a maior proporção de diagnósticos por exame de coletividade (411/38,44\%), superando até mesmo os que ocorreram por meio de encaminhamentos (275/25,64\%) e demanda espontânea (24,0\%). Padrão semelhante pode ser percebido no ano seguinte, 2005, que registrou o segundo maior coeficiente de detecção do período (109,5/100 mil habitantes na população geral). O principal modo de detecção foram os exames de coletividade $(385 / 36 \%$ dos diagnósticos), seguido de demanda espontânea (357/33,38\%) e encaminhamentos (21,0\%). Nos maiores de 15 anos, as taxas seguem um padrão muito similar às da população geral.

Quanto aos menores de 15 anos, o coeficiente de detecção mais alto foi de 31,97 casos/100 mil habitantes, considerado hiperendêmico, como quase todos os demais anos estudados. Houve um aumento no coeficiente de 3,19 para 16,3/100 mil habitantes entre os anos de 2014 e 2015, o que representou um salto para níveis hiperendêmicos.

Do total de casos novos de hanseníase ocorridos no município foram georreferenciados apenas os residentes na zona urbana (983/91,96\%) da cidade sede.

Em relação à distribuição espacial dos casos, observou-se que os bairros mais acometidos foram aqueles localizados, respectivamente, na porção Sul da cidade, onde se destacam os bairros Tancredo Neves III (159/14,82\%) e Tancredo Neves II (148/13,84\%), no Centro (105/9,82\%) e no Bairro do Perpétuo Socorro (77/7,20\%), localizados na porção Insular (Norte) de Paulo Afonso, que assumiram a $3^{\text {a }}$ e a $4^{\underline{a}}$ posição em número de casos novos de hanseníase (Figura 1).

Figura 1 - Distribuição dos casos novos de hanseníase residentes na cidade de Paulo Afonso
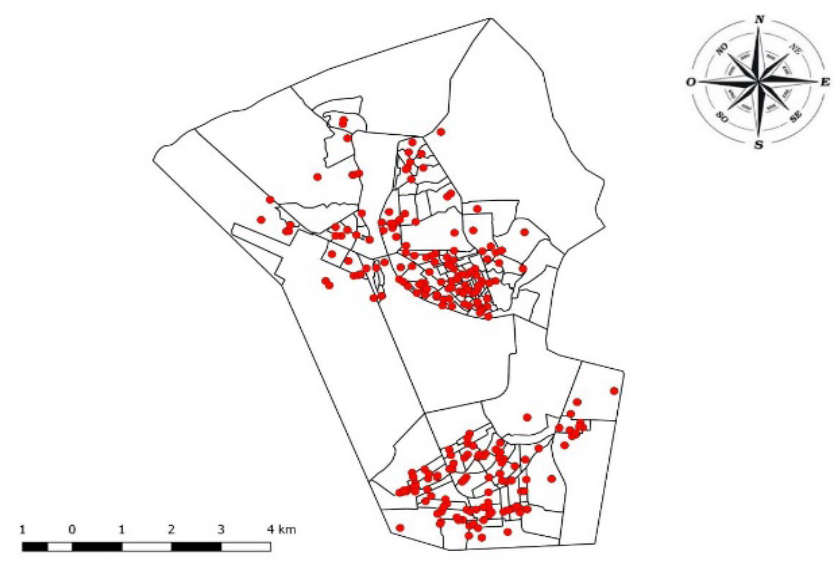

Fonte: Elaboração própria.

A distribuição dos casos novos de hanseníase nos bairros mais endêmicos evidencia a presença de pelo menos dois clusters: um localizado na porção Sul, que apresenta maior população e aonde os coeficientes de detecção são os mais elevados, e outro localizado na região Insular da cidade, acometendo principalmente a área que correspondia, no passado, à Vila Poty (Figura 2). 
Figura 2 - Clusters espaciais dos casos novos de hanseníase residentes na cidade de Paulo Afonso
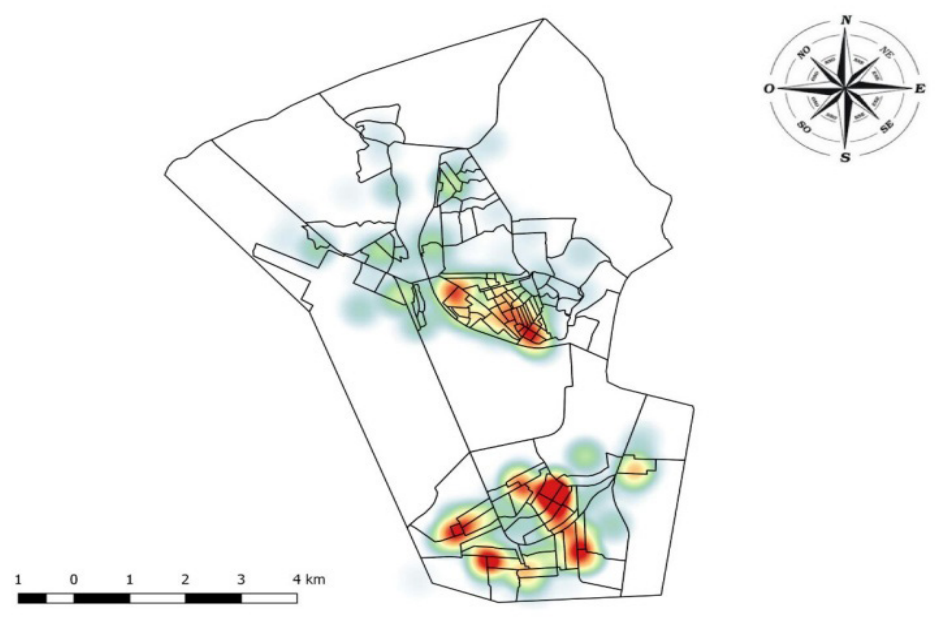

Fonte: Elaboração própria.

\section{Discussão}

O estudo revelou que os casos novos de hanseníase residentes em Paulo Afonso possuíam as características sociodemográficas de acordo com o perfil brasileiro em relação à maioria das variáveis analisadas, variação nos coeficientes de detecção entre os anos e espacialização com distribuição heterogênea e focalizada nos bairros mais periféricos.

Observou-se que a maioria dos casos novos de hanseníase predominou no sexo feminino $(57,16 \%)$, similar ao encontrado em outros estudos realizados no estado da Bahia ${ }^{(15)}$ e no Brasil $^{(8)}$. Entretanto, há uma tendência de redução de casos em mulheres na Bahia ${ }^{(16)}$. Essa maior ocorrência em mulheres pode estar relacionada ao fato de que elas tendem a se preocupar mais com a própria saúde e serem menos resistentes para procurar atendimento com profissionais da saúde ${ }^{(11)}$, assim como pode também estar relacionada ao fato de as mulheres estarem expondo-se mais, ao atravessarem a barreira domiciliar em busca do próprio espaço no mercado de trabalho ${ }^{(17)}$.

A raça/cor parda $(66,92 \%)$ foi mais frequente nas notificações, concordando com o que foi apresentado em outros estudos ${ }^{(8,10)}$. Estudo aponta que essa ocorrência deve-se ao fato de que há predominância dessa população na região Nordeste do país ${ }^{(15)}$, além de que são bastante vulneráveis as desigualdades em diversos aspectos sociodemográficos, em decorrência do contexto histórico da população negra no Brasil.

Em relação à escolaridade, a maioria dos casos novos de hanseníase possui apenas o ensino fundamental $(73,87 \%)$, dado em acordo com outros trabalhos ${ }^{(8,10)}$. Essa característica, além de outros aspectos, pode ser mais encontrada pelo fato de essa população não ter conhecimento acerca dos métodos de prevenção e dos sinais clínicos que surgem no início dessa enfermidade, e não ter discernimento suficiente em relação ao autocuidado $^{(15)}$.

O estudo evidenciou maior ocorrência de casos novos na zona urbana (93,97\%), semelhante ao encontrado em outros estudos realizados no Brasil $^{(8,15)}$. Considerando a migração do interior rural para a área urbana, estima-se que essa população tenha levado consigo as mesmas características precárias socioeconômicas, ao se deslocarem para bairros pobres e com aglomeração populacional. A literatura afirma que esse fato pode ter favorecido a disseminação da doença $^{(15)}$.

Em Paulo Afonso, percebeu-se que, na maioria dos casos novos de hanseníase, a idade apresentava estar acima de 15 anos (92,50\%),

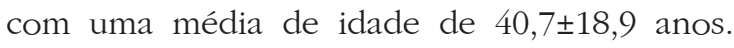
Em geral, esses indivíduos fazem parte de uma população economicamente ativa, conforme 
apresentado também por outros autores ${ }^{(10,15,17)}$. Em decorrência de a hanseníase ter um período de incubação longo, isto é, demora entre 3 a 5 anos para que o agente etiológico instale-se no organismo do hospedeiro e comece a manifestar os sinais e sintomas característicos da doença, atribui-se a essa enfermidade a denominação de doença de adulto ${ }^{(18)}$.

Já em relação aos casos novos em Paulo Afonso, a faixa etária de 15 anos constituiu 8\% das notificações. Este resultado foi equivalente ao achado em outro município do Vale do São Francisco, que registrou 7,94\% do total de casos novos diagnosticados ${ }^{(19)}$. Estudos revelam que esses jovens são atingidos cada vez mais, devido ao alto número de casos da doença na população. Este é, portanto, um indicador de uma área endêmica, representando foco de transmissão ativa e recente, visto que o período de incubação não é obedecido em decorrência da limitação funcional e da fragilidade nessa faixa etária ${ }^{(18)}$.

Em relação às incapacidades físicas, o estudo revelou maior frequência de casos novos que apresentavam algum grau de GIF no diagnóstico e tratamento. Apesar de conhecida tendência de elevação das GIF na Bahia ${ }^{(10)}$, os achados deste estudo destacaram-se quando comparados com a literatura ${ }^{(10,15,18)}$. O retardo do tratamento pode ser uma das possibilidades de explicação dessa situação, pois a presença de GIF, principalmente a GIF 2, não ocorre nos estágios iniciais da doença. Por isso, pode ser um marcador indireto de magnitude da doença, e da qualidade e efetividade da assistência prestada ${ }^{(20)}$. Um fato que chama a atenção é a frequência razoável (26,94\%) de casos não avaliados na alta, principalmente em um cenário de elevado quantitativo de GIF 1 e 2. Isso evidencia a fragilidade das equipes de saúde quanto ao manejo clínico e limita o conhecimento sobre as condições dessa pessoa no transcurso final do tratamento e no pós-alta.

A comparação do coeficiente de detecção de Paulo Afonso (28,73 casos por 100.000 habitantes) em 2014 com os coeficientes da Bahia (17,4 casos por 100.000 habitantes) e do Brasil (15,3 casos por 100.000 habitantes), nesse mesmo ano, mostrou que o município é classificado como tendo endemia muito alta (entre $20,00$ a 39,99/100 mil habitantes $)^{(10,14)}$.

É relevante o fato de Paulo Afonso ter apresentado um processo de urbanização desigual desde o início de sua história. Se, por um lado, o antigo acampamento planejado da "Cidade Jardim" beneficiou um grupo seleto de trabalhadores da Usina, constituído por engenheiros e outros profissionais técnicos, com casas de alvenaria e ótimas condições de moradia, por outro, criou enormes contrastes com as insalubres condições de moradia dos milhares de imigrantes que chegavam à procura de trabalho e se empilhavam espontaneamente ao redor do acampamento, formando a Antiga Vila Poty. Com a democratização, veio a queda do muro material que separava as duas regiões da cidade. Contudo, recentemente, o desenvolvimento de novos aglomerados urbanos na parte Sul e Noroeste tendem a reproduzir, com outras nuances, a lógica existente nos primeiros anos da cidade ${ }^{(21)}$.

Neste contexto, ao observar a distribuição dos casos novos de hanseníase notificados no município de Paulo Afonso entre os anos de 2000 e 2015, constatou-se que a localização desses segue uma lógica de distribuição espacial diretamente relacionada com as condições socioeconômicas das áreas acometidas ${ }^{(14)}$. Estudos realizados no Brasil e em outros países apontam que determinados contextos, envolvendo características de moradia, hábitos de agregação familiar e social, fluxo de pessoas incentivado por economia ou cultura, podem concorrer para a manutenção do Mycobacterium leprae circulante ${ }^{(22)}$.

A identificação de clusters envolvendo principalmente os bairros Tancredo Neves e os bairros onde outrora se localizava a Vila Poty, como é o caso do Centro, parecem sinalizar para maior transmissão da hanseníase nessas áreas. Isto ressalta a relevância da compreensão da organização do espaço geográfico local na manutenção da cadeia de transmissão e na dinâmica da hanseníase ao longo dos anos ${ }^{(23)}$. Entretanto, o menor número de casos em determinados bairros, especialmente marginais às áreas mais endêmicas, pode sugerir a necessidade de intensificação de ações voltadas para diagnóstico e 
tratamento mais precoces de doentes ${ }^{(24-26)}$. Afinal, estudo explica que a prevenção, o tratamento e a melhor assistência em saúde em áreas comprometidas concorrem para menor exposição da população ao bacilo ${ }^{(27)}$.

A maior concentração de casos envolvendo determinados bairros das porções Sul, Norte e Noroeste de Paulo Afonso evidencia que o histórico processo de segregação socioespacial vivenciado na sede do município concorreu para uma distribuição heterogênea da hanseníase entre os anos de 2000-2015, afetando majoritariamente as regiões mais pobres da cidade ${ }^{(28)}$.

As limitações encontradas no presente estudo devem-se ao fato de que nem todos os dados da ficha de notificação estavam preenchidos devidamente. Isso impediu a análise fidedigna acerca da caracterização da doença. Em decorrência disso, 12,44\% não puderam ser georreferenciados devido à incompletude das informações dos campos destinados aos endereços nas fichas de notificação. Além disso, a utilização de dados secundários restringiu a quantidade de variáveis sociodemográficas investigadas. Se, neste estudo, fosse aplicado um questionário, ter-se-ia a possibilidade de investigar outras variáveis que não constavam na ficha de notificação.

\section{Conclusão}

O estudo mostrou que Paulo Afonso, apesar do declínio do número de casos ao longo dos anos (2000-2015), ainda apresenta marcante presença de pacientes multibacilares e com grau de incapacidade no momento do diagnóstico. Tal situação, ao afetar mais mulheres e indivíduos competentes, causa prejuízos sociais e econômicos diversos, na medida em que atinge principalmente adultos em idade economicamente ativa.

Tal realidade desperta a atenção para a manutenção da cadeia de transmissão e para o maior risco de incapacidades e deformidades devido ao diagnóstico e tratamento tardios de uma parcela significativa de pacientes. Apesar de uma oscilação descendente do número de casos ao longo dos anos (2000-2015), ainda é marcante o número de pacientes multibacilares.
O estudo, ao apresentar os bairros com maior risco de adoecimento e a elevada frequência de casos que já chegavam com algum GIF, reforça a necessidade de melhor qualificação dos profissionais de saúde, de intensificação da vigilância epidemiológica e descentralização de ações a nível municipal, bem como a garantia de atenção integral à pessoa com hanseníase e/ou suas sequelas.

Ao mesmo tempo, o estudo abre espaço para futuras pesquisas, ao suscitar questionamentos como: Qual a relação entre o ritmo da urbanização de Paulo Afonso e o aumento do número de casos de hanseníase? Qual o papel do componente migratório na manutenção da cadeia de transmissão da hanseníase no município?

Diante do exposto, espera-se que os resultados deste trabalho, associados com outros estudos epidemiológicos, possibilitem maior compreensão da dinâmica da distribuição espacial da hanseníase na cidade de Paulo Afonso, colaborando para ações de educação, prevenção e controle direcionadas para as áreas de maior risco de infecção pela hanseníase.

\section{Colaborações:}

1 - concepção, projeto, análise e interpretação dos dados: Yasmin Pereira Azevedo, Vitor Augusto da Silva Bispo, Ronaldo Inácio de Oliveira, Bruno Bezerra Gondim, Sélton Diniz dos Santos, Márcio Santos da Natividade e Joilda Silva Nery;

2 - redação do artigo e revisão crítica relevante do conteúdo intelectual: Yasmin Pereira Azevedo, Vitor Augusto da Silva Bispo, Ronaldo Inácio de Oliveira, Bruno Bezerra Gondim, Sélton Diniz dos Santos, Márcio Santos da Natividade e Joilda Silva Nery;

3 - aprovação final da versão a ser publicada: Sélton Diniz dos Santos, Márcio Santos da Natividade e Joilda Silva Nery.

\section{Referências}

1. World Health Organization. Guidelines for the Diagnosis, Treatment and Prevention of Leprosy [Internet]. Genève; 2018 [cited 2019 Jun 15]. 
Available from: https://apps.who.int/iris/bitstream/ handle/10665/274127/9789290226383-eng. pdf?sequence $=10 \&$ isAllowed $=\mathrm{y}$

2. Nath I, Saini C, Valluri VL. Immunology of leprosy and diagnostic challenges. Clin Dermatol. 2015 Jan;33(1):90-8. DOI: 10.1016/j. clindermatol.2014.07.005

3. Brasil. Ministério da Saúde. Guia prático sobre a hanseníase [Internet]. Brasília (DF); 2017 [cited 2019 Jun 15]. Available from: https://portalarquivos2. saude.gov.br/images/pdf/2017/novembro/22/ Guia-Pratico-de-Hanseniase-WEB.pdf

4. Brasil. Ministério da Saúde. Caracterização da situação epidemiológica da hanseníase e diferenças por sexo, Brasil, 2012-2016. Bol Epidemiol [Internet] 2018 [cited 2020 Apr 12];49(4):1-10. Available from: https://www.saude. gov.br/images/pdf/2018/janeiro/31/2018-004Hanseniase-publicacao.pdf

5. Brasil. Ministério da Saúde. Portaria $n^{\circ}$ 1.073, de 26 de setembro de 2000. Dispõe sobre as instruções normativas destinadas a orientar o desenvolvimento das ações de controle e de eliminação da hanseníase em todo o Território Nacional, com ênfase na atenção básica de saúde, as quais regulamentam as diretrizes estabelecidas pela Portaria nº 816, de 26 de julho de 2000 [Internet]. Brasília (DF); 2000 [cited 2020 Apr 20]. Available from: http://www.credesh.ufu.br/ sites/credesh.hc.ufu.br/arquivos/Portaria_1073. GM_26.09.pdf

6. Aquino A, Medina MG, Nunes CA, Souza MF. Estratégia de saúde da família e reordenamento do sistema de serviços de saúde. In: Paim JS, Almeida-Filho N. Saúde Coletiva: Teoria e Prática. Rio de Janeiro: MedBook; 2014. p. 353-70.

7. Brasil. Ministério da Saúde. Portaria no 816, de 27 de julho de 2000. Aprova as diretrizes, a seguir apresentadas, destinadas a orientar, em todos os níveis do Sistema Único de Saúde SUS -, as medidas de prevenção, diagnóstico, tratamento e controle de hanseníase no País, que serão coordenadas, em nível nacional, pela Área Técnica de Dermatologia Sanitária da Secretaria de Políticas de Saúde deste Ministério [Internet]. Brasília (DF); 2000 [cited 2020 Feb 1]. Available: from: http://www.credesh.ufu.br/ sites/credesh.hc.ufu.br/arquivos/PORTARIA\%20 N816\%20GM\%20DE\%2026\%20DE\%20JULHO\%20 DE\%202000.pdf

8. Nery JS, Ramond A, Pescarini JM, Alves A, Strina A, Ichihara MY, et al. Socioeconomic determinants of leprosy new case detection in the 100 Million Brazilian Cohort: a population-based linkage study. Lancet Glob Health. 2019 Sep;7(9):1226-36. DOI: htt10.1016/S2214-109X(19)30260-8

9. Bahia. Secretaria Estadual de Saúde do Estado da Bahia. Boletim Epidemiológico de Hanseníase [Internet]. 2018 [cited 2019 Jan 15]. Available from: http://www.saude.ba.gov.br/wpcontent/uploads/2017/11/boletimEpidemiogico HanseniaseAgo2018_n\%C2\%BA01.pdf

10. Souza EA, Ferreira AF, Boigny $\mathrm{RN}$, Alencar $\mathrm{CH}$, Heukelbach J, Martins-Melo FR, et al. Hanseníase e gênero no Brasil: tendências em área endêmica da região Nordeste 2001-2014. Rev Saude Publica. 2018 Feb;52:20. DOI: 10.11606/ s1518-8787.2018052000335

11. Brasil. Ministério da Saúde. Portaria 2.556, de 28 de outubro de 2011. Estabelece mecanismo de repasse financeiro do Fundo Nacional de Saúde aos Fundos de Saúde Estaduais, do Distrito Federal e Municipais, por meio do Piso Variável de Vigilância e Promoção da Saúde, para implantação, implementação e fortalecimento da Vigilância Epidemiológica de Hanseníase, Tracoma, Esquistossomose e Geohelmintíases [Internet]. Brasília (DF); 2011 [cited 2020 Jul 2]. Available from: http://bvsms.saude.gov.br/bvs/saudelegis/ gm/2011/prt2556_28_10_2011.html

12. Rodrigues RN, Leano HAM, Bueno IC, Araújo KMFA, Lana FCF. Áreas de alto risco de hanseníase no Brasil, período 2001-2015. Rev Bras Enferm. 2020 Apr;73(3):e20180583. DOI: 10.1590/0034-7167-2018-0583

13. Instituto Brasileiro de Geografia e Estatística. Coordenação de População e Indicadores Sociais. Estimativa da população residente [Internet]. Rio de Janeiro; 2017 [cited 2017 Jul 1]. Available from: www.ibge.gov.br

14. Brasil. Ministério da Saúde. Portaria no 149 , de 3 de fevereiro de 2016. Aprova as Diretrizes para Vigilância, Atenção e Eliminação da Hanseníase como Problema de Saúde Pública, com a finalidade de orientar os gestores e os profissionais dos serviços de saúde [Internet]. Brasília (DF); 2011 [cited 2020 Jan 17]. Available from: http:// bvsms.saude.gov.br/bvs/saudelegis/gm/2016/ prt0149_04_02_2016.html

15. Silva MEGC, Souza CDF, Silva SPC, Costa FM, Carmo RF. Epidemiological aspects of leprosy in Juazeiro-BA, from 2002 to 2012. An Bras Dermatol. 2015 Nov/Dec;90(6):799-805. DOI: 10.1590/ abd1806-4841.201533963 
16. Souza CDF, Luna CF, Magalhães MAFM. Leprosy transmission in Bahia, 2001-2015: modeling based on Joinpoint regression and spatial scan statistics. Epidemiol Serv Saúde. 2019;28(1):e2018065 DOI: 10.5123/s1679-4974201 9000100015

17. Lobato DC, Neves DCO, Xavier MB. Avaliação das ações da vigilância de contatos domiciliares de pacientes com hanseníase no Município de Igarapé-Açu, Estado do Pará, Brasil. Rev Pan-Amaz Saúde [Internet]. 2016 [cited 2019 Mar 4];7(1):45-53. Available from: http:// scielo.iec.gov.br/scielo.php?script $=$ sci_ abstract\&pid=\$2176-62232016000100006\&lng=pt\& nrm $=$ iso

18. Monteiro MJSD, Santos GM, Barreto MTS, Silva RVS, Jesus RLR, Silva HJN. Perfil epidemiológico de casos de hanseníase em um estado do Nordeste brasileiro. Rev Aten Saude. 2017 Out/Dez;15(54):21-8. DOI: 10.13037/ras. vol15n54.4766

19. Luna ICF, Moura LTR, Vieira MCA. Perfil clínico-epidemiológico da hanseníase em menores de 15 anos no município de Juazeiro-BA. Rev bras promoc saúde. 2013 abr/jun;26(2):208-15. DOI: $10.5020 / 18061230.2013$

20. Oliveira KS, Souza J, Zilly A, Silva-Sobrinho RA. Avaliação dos indicadores epidemiológicos e operacionais para a hanseníase em municípios prioritários no estado do Paraná, 2001 a 2010. Epidemiol Serv Saude. 2015;24(3):507-16. DOI: 10.5123/S1679-49742015000300016

21. Prefeitura Municipal de Paulo Afonso. Plano Diretor de Desenvolvimento Urbano e Ambiental de Paulo Afonso: Leitura da Realidade Local. Paulo Afonso (BA): 2016.

22. Diana NJL. Commentary: Leprosy and poverty. Int J Epidemiol. 2004 Apr;33(2):269-70. DOI: 10.1093/ije/dyh115
23. Barbosa DRM, Almeida MG, Santos AG. Características epidemiológicas e espaciais da hanseníase no Estado do Maranhão, Brasil, 2001-2012. Medicina (Ribeirao Preto). 2014;47(4):347-56. DOI: 10.11606/issn.2176-7262. v47i4p347-356

24. Amaral EP, Lana FCF. Análise espacial da Hanseníase na microrregião de Almenara, MG, Brasil. Rev bras enferm. 2008;61(spe):701-7. DOI: S0034-71672008000700008

25. Ramos ACV, Yamamura M, Arroyo LH, Popolin MP, Chiaravalloti Neto F, Palha PF, et al. Spatial clustering and local risk of leprosy in São Paulo, Brazil. PLoS Negl Trop Dis. 2017;11(2):e0005381. DOI: 10.1371/journal.pntd.0005381

26. Monteiro LD, Martins-Melo FR, Brito AL, Alencar $\mathrm{CH}$, Heukelbach J. Spatial patterns of leprosy in a hyperendemic state in Northern Brazil, 2001-2012. Rev Saúde Pública. 2015;49(84). DOI: 10.1590/S0034-8910.2015049005866

27. Freitas LRS, Duarte EC, Garcia LP. Análise da situação epidemiológica da hanseníase em uma área endêmica no Brasil: distribuição espacial dos períodos 2001 - 2003 e 2010 - 2012. Rev bras epidemiol. 2017 Oct/Dec;20(4):702-13. DOI: 10.1590/1980-5497201700040012

28. Sampaio PB, Rossi TL, Cerutti Junior C, Zandonade E. Spatial analysis of new cases of leprosy in the State of Espírito Santo, Brazil, between 2004 and 2009. Rev Soc Bras Med Trop. 2012 May/Jun;45(3):380-4. DOI: 10.1590/ S0037-86822012000300019

Recebido: 9 de julho de 2020

Aprovado: 20 de agosto de 2020

Publicado: 24 de novembro de 2020

A Revista Baiana de Enfermagem utiliza a Licença Creative Commons - Atribuição-NãoComercial 4.0 Internacional. https://creativecommons.org/licenses/by-nc/4.0/

Este artigo é de acesso aberto distribuído sob os termos da Licença Creative Commons (CC BY-NC). Esta licença permite que outros remixem, adaptem e criem a partir do seu trabalho para fins não comerciais. Embora os novos trabalhos tenham de lhe atribuir o devido crédito e não possam ser usados para fins comerciais, os usuários não têm de licenciar esses trabalhos derivados sob os mesmos termos. 